\title{
Is Ovarian Cancer Prevention Currently Still a recommendation of Our Grandparents?
}

\section{A prevenção do câncer de ovário ainda é uma recomendação de nossos avós?}

\author{
Millena Prata Jammal ${ }^{1}$ Cid Almeida de Lima ${ }^{1}$ Eddie Fernando Candido Murta ${ }^{1}$ \\ Rosekeila Simões Nomelini ${ }^{1}$ \\ ${ }^{1}$ Research Institute of Oncology (IPON), Discipline of Gynecology and \\ Obstetrics; Universidade Federal doTriângulo Mineiro, Uberaba, MG, Brazil \\ Rev Bras Ginecol Obstet 2017;39:676-685. \\ Address for correspondence Prof. Rosekeila Simões Nomelini, PhD, \\ Av. Getúlio Guaritá, 214, 38025-440, Uberaba, MG, Brazil \\ (e-mail: rosekeila@terra.com.br; \\ rosekeila.nomelini@pesquisador.cnpq.br).
}
Abstract
Keywords
- ovarian neoplasms
- primary prevention
- oophorectomy
- salpingectomy
- lifestyle

\section{Resumo}

Palavras-chave

- neoplasias ováricas

- prevenção primária

- ovariectomia

- salpingectomia

- estilo de vida
Ovarian cancer is the leading cause of death among gynecologic tumors because in most of the cases (75\%), the disease is diagnosed in advanced stages. Screening methods are not available since the disease is rare, and the tested methods, such as ultrasound and CA125, were not able to decrease the mortality rate for this type of cancer. This article discusses the main risk factors for ovarian cancer, and the potential clinical and surgical strategies for the prevention of this disease.

O câncer de ovário é a principal causa de morte entre os tumores ginecológicos, já que na maioria dos casos (75\%) o diagnóstico ocorre em estádios avançados. Métodos de rastreamento não estão disponíveis, já que a doença é rara, e os métodos diagnósticos, como ultrassonografia e CA 125, não são capazes de reduzir a taxa de mortalidade desse câncer. Este artigo discute os principais fatores de risco para o câncer de ovário e as possíveis estratégias clínicas e cirúrgicas para a prevenção dessa doença.

\section{Introduction}

Currently, ovarian cancer is the leading cause of death due to a gynecological tumor. This is largely due to the non-specific symptoms associated with ovarian cancer and the absence of effective screening methods. As a result, early detection of ovarian cancer is difficult ${ }^{1}$ and ovarian malignancies are predominantly diagnosed during the advanced stages of disease (that is, stages III and IV). $)^{2,3}$ As a result, patients with ovarian cancer have the lowest survival rates among patients with gynecological tumors. In 2017, it is estimated that there will be 22,440 new cases of ovarian cancer, and $\sim 14,080$

received

June 24, 2017

accepted

October 17, 2017

published online

November 27, 2017

10.1055/s-0037-1608867.

ISSN $0100-7203$. deaths, in the United States alone. Ovarian cancer currently accounts for $\sim 5 \%$ of all cancers among women and its incidence has decreased by $1.0 \%$ per year since 1992 . However, between 2005 and 2014, the death rate from ovarian cancer decreased $2.0 \%$ per year among white women, while the death rate remained stable for African-American women. Unfortunately, more women continue to die from ovarian cancer than from all other gynecologic types of tumor combined. ${ }^{4}$

The overall survival rate for patients with ovarian cancer is less than $40 \%$ within 5 years of diagnosis. Moreover, the survival rates are $95 \%, 79 \%$, and $28 \%$ for stage I, stage II, and advanced clinical stage patients, respectively. Even with

Copyright @ 2017 by Thieme Revinter

Publicações Ltda, Rio de Janeiro, Brazil

License terms

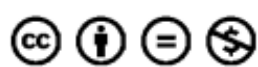


adequate treatment, $75 \%$ of ovarian cancer cases involve disease recurrence, most of which develop within the first 2 years after chemotherapy. Patients with recurrent epithelial ovarian cancer have a poor prognosis, with the median survival period being less than 2 years and the 5-year survival rate being lower than $10 \%{ }^{5}$

Primary ovarian malignant tumors can be classified into three groups: epithelial-stromal tumors, tumors of the sexual cords, and germ cell tumors. ${ }^{6}$ Among these groups, ovarian epithelial tumors account for $90 \%$ of all primary ovarian cancer cases. ${ }^{7}$ An ovarian carcinogenesis model has been established that correlates with the clinical, pathologic, and molecular features of ovarian cancer in humans. Moreover, this model can be established with Type 1 or Type II ovarian tumors. Type I tumors are slow-growing, they can develop from precursor lesions, and they include borderline serous tumors, low grade serous carcinomas, mucinous carcinoma, endometrioid carcinomas, and clear cell carcinomas. In contrast, type II tumors are highly aggressive, most are diagnosed at an advanced stage, and they include highgrade serous carcinomas, malignant mixed mesodermal tumors (carcinosarcomas), and undifferentiated tumors. ${ }^{8}$

Screenings of asymptomatic women for ovarian cancer have not been effective. Therefore, it is very important that symptoms associated with early stage ovarian cancer be identified.

To date, the symptoms that are considered suggestive of ovarian cancer include pelvic/abdominal pain, increased frequency of urination, urinary urgency, bloating, and early satiety. If these symptoms are present for less than 1 year and occur more than 12 days per month, ovarian cancer is suspected. When any 6 of these symptoms occurred more than 12 times per month and within a period less than 1 year, the associated sensitivity of ovarian cancer detection was $56.7 \%$ for early stage disease and $\mathbf{7 9 . 5 \%}$ for advanced stage disease. In addition, the specificity of ovarian cancer detection was $90 \%$ for patients over 50 years of age and $86.7 \%$ for patients younger than 50 years of age. ${ }^{9}$ It is recommended that the presence of these symptoms should be considered for a differential diagnosis of ovarian cancer. ${ }^{3}$

Despite the low sensitivity and specificity of ovarian cancer screening methods and the need to develop new early diagnostic methods, ${ }^{10}$ gynecological examination, ultrasound, and monitoring of a panel of systemic tumor markers represent approaches that are currently considered reasonable for obtaining an early diagnosis of ovarian neoplasia. ${ }^{11}$

There are several theories regarding carcinogenesis in ovarian epithelial tissue. Some conditions are involved with increasing or reducing the risk. The most consistent risk factors are related to reproductive aspects, such as nulliparity, early menarche and late menopause. ${ }^{12}$

Approximately $5-10 \%$ of all breast and ovarian cancer cases reflect hereditary genetic defects, mainly in two breast and ovary cancer genes, BRCA1 (breast cancer 1) and BRCA2 (breast cancer 2). ${ }^{13}$ Both genes encode large proteins that play critical roles in the DNA repair pathway. ${ }^{14}$ Mutations in BRCA1 and BRCA2 predispose the hereditary syndrome of breast and ovarian cancer characterized by multiple family members affected with breast or ovarian cancer, bilateral breast cancer, and other cancers in family members, including prostate, pancreas, and male breast cancer. ${ }^{15,16}$

Other factors, such as poor diet that leads to overweight or obesity, sedentary lifestyle, alcohol consumption and smoking, are studied factors implicated in the possible increase in the rate of disease development. ${ }^{17}$

The main reasons for the occurrence of ovarian cancer are still unclear. However, lifestyle factors are also important in the etiology of this disease. Current evidence suggests that the risk of ovarian cancer can be reduced with the practice of various healthy habits that are often advocated by grandparents, specifically, a good diet that includes a minimal number of non-processed foods, regular physical exercise, and avoiding smoking, prolonged hormone replacement therapy, and obesity. It is also proposed that the detection of women at high risk for ovarian cancer should be evaluated for prophylactic salpingo-oophorectomy. ${ }^{18}$

\section{Methods}

A search for the following keywords was conducted in the PubMed database: "ovarian cancer prevention," "risk factors," "oophorectomy," "salpingectomy," "oral contraceptives," "intratubal cytology," and "lifestyle." All of the articles identified were published in English between January of 1999 and July of 2017. Total 97 articles were selected for further examination.

\section{Results}

\section{Risk Factors}

Known risk factors for ovarian cancer include age, obesity, reproductive history, family history, hormone replacement therapy, and smoking (-Fig. 1). ${ }^{19}$ Conversely, factors which limit or reduce the risk of ovarian cancer include: inhibition or arrest of normal ovulation due to pregnancy or contraception, an extended lactation period, multiparity, ${ }^{20}$ and prior surgery such as fimbriectomy, salpingectomy, or salpingo-oophorectomy. Moreover, the latter has been associated with a 75-96\% reduction in the risk of ovarian cancer. ${ }^{21,22}$

In the literature, procedures have been described that may prevent the occurrence of ovarian cancer. However, there are some limitations to these procedures, such as there being no prospective studies that demonstrate the efficacy of oral contraception or bilateral salpingo-oophorectomy in patients with unknown factors for ovarian cancer. ${ }^{23}$ In the studies that have shown a reduced risk of ovarian cancer following bilateral salpingo-oophorectomy, the surgeries were generally reserved for women at high risk of developing ovarian cancer, such as those with hereditary susceptibility to ovarian cancer. In contrast, women carrying BRCA1 or BRCA2 mutations have exhibited a $90 \%$ reduction in their risk of ovarian cancer. ${ }^{24}$

\section{Clinical Strategies Related to Anovulation}

Oral Contraceptives, Reproductive and Menstrual Factors Accumulating evidence suggests that ovulation increases the levels of inflammatory agents that can lead to mutations in the DNA. For example, ovulation creates breaks in the surface of an 




Fig. 1 Risk factors associated to ovarian cancer. Abbreviations: BRCA, breast cancer.

ovary, and the subsequent healing process involves increased levels of inflammatory mediators and reactive oxygen species. Reactive oxygen species directly oxidize the DNA, and this can exponentially increase the risk of developing mutations. ${ }^{25}$ This model is consistent with the increased risk observed in patients who use ovulation-inducing drugs, and the decreased risk observed in patients who have breastfed their children, undergone pregnancy, and/or used oral contraceptives; with the latter set of conditions characterized by a reduced number of ovulatory cycles. ${ }^{26}$

The older ages associated with menarche have been found to be associated with a decreased risk of ovarian cancer (relative risk $=0.6-0.8$ ); although some studies have indicated a protective effect when menopause occurs earlier. ${ }^{27}$ The lifelong number of menstrual cycles for a woman has also been associated with ovarian cancer risk, thereby suggesting that ovulation has a role in ovarian carcinogenesis. ${ }^{28}$

Both case-control and cohort studies have demonstrated that pregnancy protects women against ovarian cancer, and that benefit increases according to the number of pregnancies. ${ }^{27}$ For example, each additional birth is predicted to reduce the likelihood of ovarian cancer by $\sim 12 \%{ }^{26}$ Accumulating evidence also indicates that the use of oral contraceptives reduces the risk of ovarian cancer, possibly due to suppression of ovulation and the direct effects of hormones on the ovary. ${ }^{29}$ Both a systematic review and a meta-analysis of 24 studies of case-controls and cohorts showed a significant reduction in the incidence of ovarian cancer in women who used oral contraceptives compared with those who did not. ${ }^{27}$ In addition, a significant duration-response relationship was observed, with a reduction in ovarian cancer risk $>50 \%$ associated with women who used oral contraceptives for 10 years or more. ${ }^{30}$

The use of oral contraceptives in patients without a known risk for ovarian cancer has also been associated with a 22-56\% decrease in risk of the disease. However, the minimum optimal duration of contraception use would be 5 years. ${ }^{31}$ In a casecontrol study of women with mutations in the BRCA1 or BRCA2, the use of oral contraceptives led to a $50 \%$ reduced risk of ovarian cancer. $^{32}$
More recently, factors that regulate the availability of sex steroids and their importance in the pathophysiological aspects of female reproductive cancers have been examined. Sexual steroid hormones (such as estradiol, progesterone, and testosterone) are involved in the etiology of ovarian cancer, and changes in these hormones have been observed when reproductive patterns exhibit alterations as well as when there is development of an ovarian cancer. For example, the use of oral steroids may increase the risk of ovarian cancer in the same way that hormone replacement therapy does, due to changes in the levels of estradiol and progesterone that occur during the menstrual cycle in premenopausal women. The high androgen levels in the pre- and postmenopausal stages correlate with the increased risk of ovarian cancer initiation. During menopause, the incidence of ovarian cancer is considerably increased because the androgens are the main steroid source produced by the ovaries. ${ }^{33}$

\section{Familial Predisposition}

\section{BRCA1 and BRCA2}

Approximately $10 \%$ of ovarian cancer patients have a genetic or familial predisposition that may increase their risk of developing the disease. ${ }^{34}$ The BRCA1 and BRCA2 are tumor suppressor genes that are located on chromosomes $17 q 21$ and 13q12-13, respectively. ${ }^{35}$ Germline mutations in these genes can be inherited in an autosomal dominant manner and significantly increase the risks of breast cancer and ovarian cancer. Moreover, patients with invasive ovarian cancer have an $11.7 \%$ chance of carrying a germline mutation in these genes. ${ }^{18}$

Women who have deleterious mutations in BRCA1 or $B R C A 2$, or whose mother or sister have genetic mutations in these genes, represent a subgroup of individuals with an increased likelihood of developing ovarian cancer. ${ }^{36}$ It has been observed that a woman with BRCA gene mutations has a $20 \%$ chance of developing breast cancer before the age of 40 . This risk increases to $37 \%$ after the age of 50 , to $55 \%$ up to the age of 60 , and is more than $70 \%$ by the age of $70 .{ }^{37}$ For ovarian cancer, the risk associated with BRCA1 mutations ranges 
from $45-60 \%$, and from $11-35 \%$ for $B R C A 2$ mutations. ${ }^{38}$ Ovarian carcinomas associated with mutations in the $B R C A$ gene are also considerably more aggressive than sporadic tumors. However, sensitivity to platinum-based treatments is high. In addition, a more favorable prognosis has been reported for patients carrying BRCA2 mutations compared with patients with sporadic ovarian cancer, and this cancer does not occur in women with BRCA1 mutations. ${ }^{39}$

When the epithelium at the surface of an ovary is disrupted during ovulation, tubal epithelial cells of the fimbriae of the fallopian tube can move and implant in the ovary, thereby forming an inclusion cyst. It is possible that a serous carcinoma can subsequently develop. ${ }^{8}$ In women with a genetic predisposition to ovarian cancer, as well as those without known mutations in the BRCA gene, ${ }^{40}$ small foci of tubal carcinomas can possibly invade the ovarian epithelium.

\section{Surgical Prophylaxis}

\section{Oophorectomy and Salpingectomy}

The prophylactic oophorectomy has been recommended for women with known BRCA1 or BRCA2 and who do not wish to get pregnant. ${ }^{41}$ The risk-reducing salpingo-oophorectomy surgery involves removal of the ovaries and fallopian tubes before the occurrence of a clinically apparent cancer. An additional benefit of this surgery is the apparent reduction in the risk of breast cancer, presumably because of the ablation of ovarian hormones. ${ }^{42}$

Women who carry mutations in the BRCA1 or BRCA2 genes are often advised to consider prophylactic bilateral salpingooophorectomy surgery ${ }^{42}$ after the age of 35 or after they no longer want to become pregnant. This procedure can reduce the risk of ovarian cancer and the risk of breast cancer due to ablation of ovarian hormone production. ${ }^{43,44}$

Over the last decade, a model has been proposed for ovarian carcinogenesis that starts in the fallopian tube. ${ }^{8}$ It was suggested that bilateral salpingectomy could prevent this process by removing the potential tissue source for ovarian cancer and the risks of surgical menopause would be avoided. For patients at high risk for ovarian cancer, this method has been proposed in clinical trials. ${ }^{45}$ In addition, the Society of Gynecologic Oncology (SGO) published their recommendation in 2013 that bilateral salpingectomy should be considered at the time of hysterectomy. ${ }^{46}$ It is predicted that this approach could reduce the risk of ovarian cancer by $20-40 \%$ over the next 20 years. ${ }^{47,48}$ In another case-control study, tubal ligation decreased the incidence of disease in $30 \%$ of the cases examined. ${ }^{49}$ Accumulating evidence indicates that the most common and lethal form of ovarian cancer, the high-grade serous subtype, originates in the distal fallopian tube. Consequently, it is recommended that bilateral salpingectomy be performed during gynecological surgeries, particularly during hysterectomy and tubal sterilization procedures, and for this reason, it is called opportunistic bilateral salpingectomy. ${ }^{50}$ The American Congress of Obstetricians and Gynecologists has issued a statement endorsing the recommendation that surgeons and patients discuss fallopian tube removal during hysterectomy without oophorectomy, and that bilateral salpingectomy should be presented when counseling women about methods of laparoscopic sterilization. ${ }^{51}$

\section{Risks of Surgery}

In premenopausal women, salpingo-oophorectomy induces a sudden onset of menopause. As a result, women can experience severe hot flashes, vaginal dryness, sexual dysfunction, sleep disturbances, and cognitive impairments following surgery, and these can affect the quality of life. Premature menopause is also a significant risk factor for osteoporosis and ischemic heart disease. ${ }^{43}$ Several authors have emphasized the risk of developing peritoneal carcinoma following prophylactic oophorectomy. For example, among 324 women who underwent prophylactic oophorectomy surgery at an ovarian cancer center in Buffalo, NY, 1.8\% developed peritoneal carcinoma between 1 and 27 years after the surgery. ${ }^{52}$

The salpingectomy is better at reducing risks related to early menopause. However, the safety of this surgery in regard to preservation of ovarian function is not well defined. If a salpingectomy does not interfere with ovarian blood flow, it should not adversely affect ovarian functions (that is, hormone production, ovulation, and menopause age). ${ }^{53}$ However, this remains to be confirmed.

In a recent study that evaluated 425,180 women undergoing hysterectomies between 2008 through 2013 in the United States, a $371 \%$ increase in the number of surgeries that included bilateral salpingectomy was observed. ${ }^{54}$ For these combined surgeries, no increase in the risk of blood transfusion, complications, or postoperative infections were observed compared with the women who underwent hysterectomy alone. ${ }^{54}$

Unfortunately, prospective studies to investigate the effect of patient age on salpingectomy and risk of ovarian cancer would require a long follow-up period. Moreover, residual microscopic fimbrial tissue on the ovarian surface can be found, which could decrease the impact of bilateral salpingectomy in reducing the risk of ovarian cancer. ${ }^{55}$ In the absence of these results, salpingectomies should only be performed when a suitable opportunity arises, and should not be performed only to prevent ovarian cancer. ${ }^{49}$

\section{Intratubal Cytology}

In a study published by Lum et $\mathrm{al}^{56}$, the effectiveness of cytological evaluations of fallopian tubes in patients at high risk for developing ovarian cancer was examined. Minimally invasive surgical techniques were performed to exfoliate cells of distal fallopian tubes and the proximal portion of fimbriae. When precursor lesions of ovarian cancer were found in the distal fallopian tubes, an abundance of significant cells were observed in the smears. A subset of smears also included epithelial cells of the tubes. This study demonstrated the potential for patients to be evaluated prior to surgical procedures or during more invasive interventions. During laparoscopic hysterectomy, a $6.5 \mathrm{~mm}$ hysteroscope was used and an endoscopic cytology brush could be introduced separately into each tubal ostium to reach the fimbriae end. ${ }^{56}$

When examining the samples obtained during prophylactic bilateral salpingo-oophorectomies that were performed 
in patients with BRCA1 and BRCA2 mutations, hidden cancers were found in $4-17 \%$ of the samples, with $57-100 \%$ of the cancers located in the distal portion of the fallopian tubes. Thus, the end distal fringes of the fallopian tube were identified as possible sites of origin for ovarian and peritoneal serous carcinomas. These results were encouraging given that the ability to identify serous tubal intraepithelial carcinoma at an earlier stage could prevent the rapid spread of high-grade invasive serous carcinomas and facilitate the early detection of ovarian cancer. ${ }^{56}$

\section{Lifestyle}

A relationship between ovarian cancer and lifestyle has been observed, especially regarding smoking, alcohol consumption, physical exercise, and diet. ${ }^{57}$

\section{Smoking and Alcohol Consumption}

The incidence of mucinous ovarian epithelial tumors in women with a history of smoking is well above the incidence of other histological subtypes, and it is higher than the incidence of these tumors in women who have never smoked. ${ }^{58}$ Despite these observations, however, it has not been thoroughly investigated whether smoking correlates with overall disease-free survival in patients with various histological types of ovarian epithelial cancer. To date, it is recognized that the association between smoking and patient survival differs according to the tumor stage at the time of diagnosis. In a recent study, it was observed that among women diagnosed with ovarian cancer, those who were smokers and those who had a history of smoking + had a worse survival compared with women who had never smoked. Based on these data, the authors suggest that smoking has a greater impact on survival among women with "in situ" disease than those with disseminated disease. Furthermore, cigarette was found to be a modifiable factor associated with survival and it improved disease prognosis. ${ }^{59}$

Alcohol consumption increases serum and urinary concentrations of androgens, estrogen, and other sex hormones, and it has been widely associated with an increased incidence of breast cancer. ${ }^{60}$ Epidemiological studies have also reported a direct correlation between alcohol consumption and an increased risk of developing ovarian cancer, and this correlation is dependent on the amount and duration of exposure (chronic versus acute exposure). Moreover, the effects of alcohol on ovarian tumorigenesis have been shown to be mainly caused by hormonal disorders. The most deleterious effects of chronic alcohol intake on the ovaries include alterations in estrogen and progesterone receptors, increases in estradiol levels, production of reactive oxygen species, and changes in ovary structure and size. Furthermore, ethanol and its metabolite, acetaldehyde, are capable of depleting folate levels, while also inducing DNA adduct formation and cytochrome P450-mediated reactive oxygen species generation. A DNA adduct is a segment of DNA bound to a cancer-causing chemical. It is proposed that all of these metabolic changes can be associated with the risk of tumor development. ${ }^{61}$

In contrast, studies that have evaluated the effects of alcohol consumption, from any source, on the incidence and survival of patients diagnosed with ovarian cancer remain inconsistent. Therefore, it remains to be demonstrated whether alcohol has a direct role on ovarian cancer. ${ }^{62,63}$

\section{Physical Exercise and Obesity}

Being overweight is associated with an increased risk of ovarian cancer. In a systematic review of 47 epidemiological studies of 25,157 women with ovarian cancer and 81,211 women without ovarian cancer, the relative risk for ovarian cancer with a $5 \mathrm{~kg} / \mathrm{m}^{2}$ increase in body mass index was 1.10 (95\% confidence interval, 1:07 to $1: 13$ ). ${ }^{31}$

Various studies have examined whether physical exercise can reduce obesity, reduce inflammation, and improve hormone levels and immune system function, all of which are factors involved in carcinogenesis. ${ }^{64}$ In particular, it has been investigated whether exercise intensity and frequency can be beneficial to cancer development. It has been advocated that exercising for 150 minutes/week, or 30 minute/day, 5 times per week, is advantageous. The American Cancer Society further recommends that 45 minute of exercise a day, 5 times a week, is beneficial. With respect to cancer prevention, high intensity exercise is especially recommended. ${ }^{64}$

In general, the benefits of physical exercise for health are well established. A protective effect of physical exercise is also predicted for the development of ovarian cancer as a result of better blood circulation that can directly reduce adipose tissue, thereby balancing the release of circulating estrogen and inducing a lower ovulation frequency. These effects have the potential to reduce chronic inflammation in adipose tissue as well. ${ }^{58}$

Chronic inflammation that is associated with obesity may involve continuous activation of the innate immune system, and this in turn can induce the secretion of cytokines by adipose tissue. Correspondingly, chronic inflammation associated with obesity has been linked with increased secretion of interleukin (IL)-1, IL-6 and tumor necrosis factor (TNF)- $\alpha$, which leads to a decrease in adiponectin production and an inability to store excess free fatty acids. The latter can contribute to the development of insulin resistance, type 2 diabetes mellitus, and obesity-related cardiovascular disease. In addition, a mitogenic and anti-apoptotic environment that is caused by elevated levels of insulin in obesity can accelerate a gradual accumulation of mutations, which can favor carcinogenesis. ${ }^{65}$ Aberrant production of adipokines in obese individuals, particularly involving the positive feedback loop of leptin and the negative feedback loop of adiponectin, may explain the observed association between obesity and the progression of ovarian cancer. Leptin has exhibited mitogenic and anti-apoptotic properties in various cancer cell lines and plays a role in promoting angiogenesis. On the other hand, adiponectin mediates anti-proliferative effects via induction of apoptosis. Thus, obesity can affect the survival of ovarian cancer patients via inflammatory cytokines, insulin resistance markers, and obesity-related hormones, such as estrogen, that contribute to the conversion of androgens into estrogen in adipose tissue. ${ }^{66}$

Tumor necrosis factor- $\alpha$, IL-6, and IL-1 can stimulate carcinogenesis by increasing cell proliferation, and this can lead to the activation of neoangiogenic cells. It may also 
increase the release of growth factors by endothelial cells. In association with these factors, the metabolic syndrome, as well as various cancers, have been found to be related to inflammatory conditions. Immunological alterations that can affect the behavior and prognosis of neoplasms through the interaction of immune cells originating from chronically inflamed adipose tissue should also be considered. ${ }^{65}$

According to Reid et $\mathrm{al}^{58}$, the direct effects of physical exercise on ovarian cancer have not yet been demonstrated. However, the authors acknowledge that accumulating evidence has demonstrated that physical exercise provides beneficial effects in regard to weight control, bone density, and heart disease. For sedentary women who report chronic recreational inactivity and who perform low intensity exercises, a higher incidence of ovarian cancer has been observed independent of tumor histological type or disseminated disease. ${ }^{58}$

\section{Food}

The hypothesis that some compounds from plant foods have favorable effects on the risk of ovarian cancer has been an area of active study. ${ }^{67}$

Data regarding the relationship between ovarian cancer and the intake of high-fat diets are limited and inconsistent. While some studies have demonstrated an association between ovarian epithelial cancer and a high intake of saturated fat, ${ }^{64,68-70}$ other studies have not shown this association. ${ }^{71,72}$ Intake of starchy foods and diets with high glycemic index and glycemic load have also been linked to an increased risk of ovarian cancer, although this relationship has not been confirmed. $^{73}$

An Australian study evaluated two population-based cohort and case-control studies as well as a meta-analysis, and it was observed that a greater intake of red and processed meats may be associated with an increased risk of ovarian cancer due to the high concentrations of fats in these foods. Carcinogenic heterocyclic amines and nitrous compounds are also present in red and processed meats, and these components could eventually lead to accelerated tumor development. In contrast, intake of birds or fish was associated with a possible lower risk of ovarian cancer, partly because fish is a rich source of polyunsaturated fatty acids (such as, omega-3). Meanwhile, poultry meat has a higher protein content and a lower concentration of saturated fats than red meat. Taken together, these results suggest that a diet that accommodates a reduced intake of processed meats and an increased intake of poultry and fish could reduce the risk of developing ovarian cancer. ${ }^{74}$

Generally, the intake of natural products or herbs as a supplement to cancer treatment is intended to reduce the side effects of conventional therapies and block carcinogenesis. To date, clinical data regarding the intake of natural products appears to be positive, although most studies have not included randomized clinical trials. Currently, more than half of the drugs available for treatment derived from natural products. Both derivatives from plants and anticancer agents that block cell proliferation induce apoptosis. Epidemiological studies have also shown that natural products and nutrients can be active in cancer chemoprevention. ${ }^{74}$ For example, curcumin (diferuloylmethane) is a phenolic compound derived from the Curcuma longa plant that represents a promising chemopreventive and anticancer agent. Correspondingly, a diet rich in curcumin has been shown to inversely correlate with several human malignancies. ${ }^{75,76}$ Resveratrol (3,4', 5-trihydroxytrans-stilbene) is a polyphenolic compound that is present in the skin of grapes, in red wine, peanuts, and blackberries. Resveratrol has exhibited anti-aging properties, preventative effects on cardiovascular events, and cancer protection properties. Regarding the latter, resveratrol has been shown to block cell proliferation and induce apoptosis. ${ }^{77,78}$

Flavonoids are polyphenolic plant species that have exhibited anti-allergic, anti-inflammatory, antioxidant, antimicrobial, and anticancer activities. Consequently, they are a topic of great interest. Thus, it has been demonstrated that flavonoids are able to inhibit hormone-related cancers by modulating the activity of steroid hormone receptors. ${ }^{79}$

\section{Comorbidities}

The presence of previously existing comorbidities, mainly hypertension, diabetes and its possible complications, ${ }^{80}$ are of great interest in the survival of women with ovarian cancer. These diseases may influence the prognosis directly, perhaps affecting the cellular biology of cancer or increasing the production of growth factors that influence the evolution of cancer cells as a result of prolonged exposure to hyperglycemia in patients with preexisting diabetes. In addition, they may indirectly affect the prognosis by altering patients' ability to tolerate chemotherapy or surgery. ${ }^{81,82}$

In addition, some studies suggest that the use of commonly prescribed medications for hypertension and other cardiovascular conditions, such as $\beta$-adrenergic receptor blockers, may limit the growth of ovarian tumors. This is because ovarian tumors tend to express adrenergic receptors that can bind to $\beta$-blockers and lead to decreased invasion of ovarian cancer cells in vitro, ${ }^{83,84}$ but such a claim is controversial. ${ }^{85}$

A study that analyzed pooled data from 15 studies that participated in the Ovarian Cancer Association Consortium on the relationship between history of hypertension, diabetes, their medications, and survival among patients diagnosed with invasive ovarian epithelial carcinoma. The results demonstrated that diabetes can influence the prognosis of ovarian cancer patients, affect treatment and increase the risk of death in these patients. Significant associations of mortality for hypertension were not observed. The use of $\beta$ blockers, oral antidiabetic drugs and insulin was associated with increased mortality. ${ }^{82}$ On the other hand, the literature has described that the use of metformin can be an important factor for the decrease in the risk of ovarian cancer development. ${ }^{86-88}$ Integrating the clinical profile of patients with ovarian cancer may be essential in understanding the factors related to global morbidity and mortality. ${ }^{82}$

\section{Melatonin}

Melatonin has previously been identified as a substance with immune enhancing properties and a capacity to reduce oxidative stress. More recently, antitumor properties have also been observed for melatonin. The lipophilic and hydrophilic 
properties of melatonin facilitate its access to many tissues and fluids. Moreover, depending on its production site and target organ, melatonin may act as a hormone, immuno-modulator, or as a biological modifier. To identify the role played by melatonin in apoptosis and necrosis, the ovarian cancer cell lines OVCAR-429 and PA- 1 were subjected to increasing doses of melatonin $(0,400,600$, and $800 \mu \mathrm{M})$ for $24-72$ hours. The treatment with melatonin reduced the survival and proliferation of both of the cell lines tested. Thus, melatonin could potentially be used as a therapeutic substance for the prevention or paralysis of neoplastic growth, but this remains to be confirmed. ${ }^{89,90}$

\section{Vitamin D}

Some of the traditional roles established for vitamin D include regulation of bone metabolism and homeostasis of calcium and phosphorous, and both directly affect bone health. Vitamin D also influences the normal functioning of immune cells, including $\mathrm{T}$ and $\mathrm{B}$ lymphocytes, macrophages, dendritic cells, and keratinocytes. Moreover, very low levels of vitamin D have been related to autoimmune diseases such as Crohn's disease, type 1 diabetes mellitus, multiple sclerosis, asthma, and rheumatoid arthritis. However, the importance of vitamin D action is not limited to bone health. Vitamin D has also been identified as a promising target for the treatment of diseases mediated by the immune system. It should be noted that the calcium status can also influence the effect of vitamin D and immunity. ${ }^{91}$

Intense sun exposure can be damaging to an individual's health and is largely associated with the development of cataracts, a leading cause of blindness worldwide. Excessive sun exposure is also related to the development of skin cancer and other comorbidities associated with changes in squamous cells. ${ }^{92}$ Despite these known risks, exposure to direct sunlight is necessary for the transformation of vitamin $\mathrm{D}$ into its active form in the human body, and this has benefits for bone and metabolic functions. ${ }^{93}$ Studies have also shown that vitamin $D$ reduces the risk of ovarian cancer by inhibiting the growth and apoptosis of tumor cells in culture and in animal models. ${ }^{94}$ In another study, vitamin D supplementation was associated with decreased occurrence of borderline serous ovarian tumors and endometrioid tumors, ${ }^{95}$ while higher concentrations of circulating vitamin D have been found to be inversely associated with the risk of developing ovarian cancer. ${ }^{92}$

Vitamin D and its analogues have also exhibited a capacity to suppress the invasive behavior of epithelial ovarian cancer. This observation is consistent with vitamin D activity in tumor tissue, the proliferation of tumor cells according to specific vitamin D receptors (VDRs), and the observation that compounds can regulate vitamin $\mathrm{D}$ activity in tumor tissue. An active metabolite of vitamin D, $1 \alpha, 25$-dihydroxyvitamin D3 $\left(1 \alpha, 25[\mathrm{OH}]_{2} \mathrm{D}_{3}\right.$, also known as calcitriol) may represent a potential anti-cancer agent since administration of this vitamin $D$ analogue has been shown to inhibit cell proliferation, activate apoptotic pathways, and inhibit angiogenesis by binding to VDRs. Furthermore, VDRs are present in almost all human tissues. ${ }^{96}$ Upon binding of vitamin $\mathrm{D}$ to its receptor, the receptor is activated, particularly in tissues with a high metabolic rate and high rates of cell proliferation. This function is similar to those observed in members of the p53 family, which include anti-proliferative activities mediated through induction of cell cycle arrest, senescence, and differentiation to induce apoptosis. Consequently, vitamin D is a determining factor in the autocrine suppressive regulation of various tumor types. ${ }^{97}$

To explore the chemoprevention capacity of vitamin $\mathrm{D}$, an epithelial ovarian cancer was induced by 7,12-dimethylbenz [a] anthracene (DMBA) in the presence and in the absence of vitamin D for 20 weeks. Vitamin D not only delayed the malignant transformation of epithelial cells of the ovary, but also played a chemoprotective role. In addition, no change in serum calcium concentrations were observed throughout the study, while vitamin $D$ reduced the concentration of CA125 both in serum and ascitic fluid. The biological actions of vitamin $D$ are mediated directly by VDRs, and lower levels of receptor expression and vitamin $\mathrm{D}$ are closely associated with the occurrence of ovarian cancer. The authors attribute the observed slowing of the ovarian epithelial cancer progression to overstimulation of the epithelial adhesion protein, E-cadherin, by vitamin D. In addition, the VDRs could down regulate $\beta$-catenin, thereby leading to protection of ovarian epithelial cells. ${ }^{98}$ Based on these results, the importance of vitamin $\mathrm{D}$ supplementation for patients with ovarian cancer is demonstrated.

\section{Conclusion}

Prevention of ovarian cancer remains a challenge due to our limited knowledge of its causes, difficulties associated with the modification of several established risk factors, and limited availability of early detection techniques. It is crucial to understand the role of additional factors related to the prognosis of ovarian cancer, including factors that are potentially modifiable and may contribute to change the progression of ovarian cancer and improve survival. However, it is proposed that prevention of ovarian cancer can be achieved with clinical strategies related to anovulation or surgical prophylaxis and the recommendations provided by many grandparents: we need to eat healthy, maintain physical activity, monitor weight control, and sunbathe moderately.

\section{Conflicts to Interest}

None to declare.

\section{Acknowledgments}

The authors wish to acknowledge the funding received from the Conselho Nacional de Desenvolvimento Científico e Tecnológico (CNPq, in the Portuguese acronym), Fundação de Ensino e Pesquisa de Uberaba (FUNEPU, in the Portuguese acronym) and Fundação de Amparo à Pesquisa do Estado de Minas Gerais (FAPEMIG, in the Portuguese acronym).

\section{References}

1 Jia L, Ren JM, Wang YY, et al. Inhibitory role of prohibitin in human ovarian epithelial cancer. Int J Clin Exp Pathol 2014;7(05):2247-2255

2 Jemal A, Siegel R, Xu J, Ward E. Cancer statistics, 2010. CA Cancer J Clin 2010;60(05):277-300 
3 Jelovac D, Armstrong DK. Recent progress in the diagnosis and treatment of ovarian cancer. CA Cancer J Clin 2011;61(03): 183-203

4 American Cancer Society. Cancer Facts \& Figures 2017. Atlanta, GA: ACS; 2017. https://www.cancer.org/research/cancer-facts-statistics/ all-cancer-facts-figures/cancer-facts-figures-2017.html. Accessed March 10, 2017

5 Gadducci A, Cosio S, Zola P, Landoni F, Maggino T, Sartori E. Surveillance procedures for patients treated for epithelial ovarian cancer: a review of the literature. Int J Gynecol Cancer 2007;17 (01):21-31

6 Tavassoli FA, Devilee P, Eds. Pathology and Genetics of Tumours of the Breast and Female Genital Organs. Lyon: IARC Press; 2003. (World Health Organization Classification of Tumours; Vol. 5)

7 Feng Y, He F, Wu H, et al. GOLPH3L is a novel prognostic biomarker for epithelial ovarian cancer. J Cancer 2015;6(09):893-900

8 Kurman RJ, Shih IeM. The origin and pathogenesis of epithelial ovarian cancer: a proposed unifying theory. Am J Surg Pathol 2010;34(03):433-443

9 Goff BA, Mandel LS, Drescher CW, et al. Development of an ovarian cancer symptom index: possibilities for earlier detection. Cancer 2007;109(02):221-227

10 Wang ZH, Xu CJ. Research progress of microRNA in early detection of ovarian cancer. Chin Med J (Engl) 2015;128(24):3363-3370

11 Murta EF, Nomelini RS. Early diagnosis and predictors of malignancy of adnexal masses. Curr Opin Obstet Gynecol 2006;18(01): 14-19

12 Partridge E, Kreimer AR, Greenlee RT, et al; PLCO Project Team. Results from four rounds of ovarian cancer screening in a randomized trial. Obstet Gynecol 2009;113(04):775-782

13 Claus EB, Schildkraut JM, Thompson WD, Risch NJ. The genetic attributable risk of breast and ovarian cancer. Cancer 1996;77 (11):2318-2324

14 Ciccia A, Elledge SJ. The DNA damage response: making it safe to play with knives. Mol Cell 2010;40(02):179-204

15 Petrucelli N, Daly MB, Feldman GL. Hereditary breast and ovarian cancer due to mutations in BRCA1 and BRCA2. Genet Med 2010;12 (05):245-259

16 Määttä KM, Nurminen R, Kankuri-Tammilehto M, Kallioniemi A, Laasanen S-L, Schleutker J. Germline EMSY sequence alterations in hereditary breast cancer and ovarian cancer families. BMC Cancer 2017;17(01):496

17 La Vecchia C. Ovarian cancer: epidemiology and risk factors. Eur J Cancer Prev 2017;26(01):55-62

18 Hanna L, Adams M. Prevention of ovarian cancer. Best Pract Res Clin Obstet Gynaecol 2006;20(02):339-362

19 Pylväs-Eerola M, Karihtala P, Puistola U. Preoperative serum 8hydroxydeoxyguanosine is associated with chemoresistance and is a powerful prognostic factor in endometrioid-type epithelial ovarian cancer. BMC Cancer 2015;15:493

20 Clarke-Pearson DL. Clinical practice. Screening for ovarian cancer. N Engl J Med 2009;361(02):170-177

21 Finch A, Beiner M, Lubinski J, et al; Hereditary Ovarian Cancer Clinical Study Group. Salpingo-oophorectomy and the risk of ovarian, fallopian tube, and peritoneal cancers in women with a BRCA1 or BRCA2 Mutation. JAMA 2006;296(02):185-192

22 Rebbeck TR, Lynch HT, Neuhausen SL, et al; Prevention and Observation of Surgical End Points Study Group. Prophylactic oophorectomy in carriers of BRCA1 or BRCA2 mutations. N Engl J Med 2002;346(21):1616-1622

23 Brawley OW. Ovarian cancer prevention: Time for primetime? Cancer 2015;121(13):2121-2123

24 PDQ Screening and Prevention Editorial Board. Ovarian, Fallopian Tube, And Primary Peritoneal Cancer Prevention (PDQ $\left.{ }^{\circledR}\right)$ : Health Professional Version. Jun 2017. https://www.ncbi.nlm.nih.gov/ books/NBK65921/. Accessed July 20, 2017

25 Murdoch WJ. Perturbation of sheep ovarian surface epithelial cells by ovulation: evidence for roles of progesterone and poly
(ADP-ribose) polymerase in the restoration of DNA integrity. J Endocrinol 1998;156(03):503-508

26 Modan B, Hartge P, Hirsh-Yechezkel G, et al; National Israel Ovarian Cancer Study Group. Parity, oral contraceptives, and the risk of ovarian cancer among carriers and noncarriers of a BRCA1 or BRCA2 mutation. N Engl J Med 2001;345(04):235-240

27 Titus-Ernstoff L, Perez K, Cramer DW, Harlow BL, Baron JA, Greenberg ER. Menstrual and reproductive factors in relation to ovarian cancer risk. Br J Cancer 2001;84(05):714-721

28 Pelucchi C, Galeone C, Talamini R, et al. Lifetime ovulatory cycles and ovarian cancer risk in 2 Italian case-control studies. Am J Obstet Gynecol 2007;196(01):83.e1-83.e7

29 Fathalla MF. Incessant ovulation and ovarian cancer - a hypothesis re-visited. Facts Views Vis ObGyn 2013;5(04):292-297

30 Havrilesky LJ, Moorman PG, Lowery WJ, et al. Oral contraceptive pills as primary prevention for ovarian cancer: a systematic review and meta-analysis. Obstet Gynecol 2013;122(01): 139-147

31 Collaborative Group on Epidemiological Studies of Ovarian Cancer. Ovarian cancer and body size: individual participant metaanalysis including 25,157 women with ovarian cancer from 47 epidemiological studies. PLoS Med 2012;9(04):e1001200

32 Narod SA, Risch H, Moslehi R, et al; Hereditary Ovarian Cancer Clinical Study Group. Oral contraceptives and the risk of hereditary ovarian cancer. N Engl J Med 1998;339(07):424-428

33 Chuffa LG, Lupi-Júnior LA, Costa AB, Amorim JP, Seiva FR. The role of sex hormones and steroid receptors on female reproductive cancers. Steroids 2017;118:93-108

34 Risch HA, Weiss NS, Lyon JL, Daling JR, Liff JM. Events of reproductive life and the incidence of epithelial ovarian cancer. Am J Epidemiol 1983;117(02):128-139

35 Yoshida K, Miki Y. Role of BRCA1 and BRCA2 as regulators of DNA repair, transcription, and cell cycle in response to DNA damage. Cancer Sci 2004;95(11):866-871

36 Horiuchi A, Itoh K, Shimizu M, et al. Toward understanding the natural history of ovarian carcinoma development: a clinicopathological approach. Gynecol Oncol 2003;88(03):309-317

37 Bougie O, Weberpals JI. Clinical Considerations of BRCA1- and BRCA2-mutation carriers: a review. Int J Surg Oncol 2011; 2011:374012

38 van der Kolk DM, de Bock GH, Leegte BK, et al. Penetrance of breast cancer, ovarian cancer and contralateral breast cancer in BRCA1 and BRCA2 families: high cancer incidence at older age. Breast Cancer Res Treat 2010;124(03):643-651

39 Girolimetti G, Perrone AM, Santini D, et al. BRCA-associated ovarian cancer: from molecular genetics to risk management. BioMed Res Int 2014;2014:787143

40 Przybycin CG, Kurman RJ, Ronnett BM, Shih IeM, Vang R. Are all pelvic (nonuterine) serous carcinomas of tubal origin? Am J Surg Pathol 2010;34(10):1407-1416

41 Kauff ND, Satagopan JM, Robson ME, et al. Risk-reducing salpingooophorectomy in women with a BRCA1 or BRCA2 mutation. N Engl J Med 2002;346(21):1609-1615

42 Domchek SM, Rebbeck TR. Prophylactic oophorectomy in women at increased cancer risk. Curr Opin Obstet Gynecol 2007;19(01): 27-30

43 Domchek SM, Friebel TM, Neuhausen SL, et al. Mortality after bilateral salpingo-oophorectomy in BRCA1 and BRCA2 mutation carriers: a prospective cohort study. Lancet Oncol 2006;7(03): 223-229

44 Finch A, Shaw P, Rosen B, Murphy J, Narod SA, Colgan TJ. Clinical and pathologic findings of prophylactic salpingo-oophorectomies in 159 BRCA1 and BRCA2 carriers. Gynecol Oncol 2006;100(01): 58-64

45 Greene MH, Mai PL, Schwartz PE. Does bilateral salpingectomy with ovarian retention warrant consideration as a temporary bridge to risk-reducing bilateral oophorectomy in BRCA1/2 mutation carriers? Am J Obstet Gynecol 2011;204(01):19.e1-19.e6 
46 Society of Gynecologic Oncology. SGO Clinical Practice Statement: Salpingectomy for Ovarian Cancer Prevention. Nov 2013. https:// www.sgo.org/clinical-practice/guidelines/sgo-clinical-practicestatement-salpingectomy-for-ovarian-cancer-prevention/. Accessed January 25, 2017

47 Kwon JS, McAlpine JN, Hanley GE, et al. Costs and benefits of opportunistic salpingectomy as an ovarian cancer prevention strategy. Obstet Gynecol 2015;125(02):338-345

48 Szender JB, Lele SB. Fallopian tube ligation or salpingectomy as means for reducing risk of ovarian cancer. AMA J Ethics 2015;17 (09):843-848

49 Sieh W, Salvador S, McGuire V, et al; Australian Cancer Study (Ovarian Cancer); Australian Ovarian Cancer Study Group; Ovarian Cancer Association Consortium. Tubal ligation and risk of ovarian cancer subtypes: a pooled analysis of case-control studies. Int J Epidemiol 2013;42(02):579-589

50 Ely LK, Truong M. The role of opportunistic bilateral salpingectomy vs tubal occlusion or ligation for ovarian cancer prophylaxis. J Minim Invasive Gynecol 2017;24(03):371-378

51 Committee on Gynecologic Practice. Committee opinion no. 620: Salpingectomy for ovarian cancer prevention. Obstet Gynecol 2015;125(01):279-281

52 Coukos G, Rubin SC. Prophylactic oophorectomy. Best Pract Res Clin Obstet Gynaecol 2002;16(04):597-609

53 Hanley GE, McAlpine JN, Kwon JS, Mitchell G. Opportunistic salpingectomy for ovarian cancer prevention. Gynecol Oncol Res Pract 2015;2:5

54 Hanley GE, McAlpine JN, Pearce CL, Miller D. The performance and safety of bilateral salpingectomy for ovarian cancer prevention in the United States. Am J Obstet Gynecol 2017;216(03):270.e1-270.e9

55 Gan C, Chenoy R, Chandrasekaran D, et al. Persistence of fimbrial tissue on the ovarian surface after salpingectomy. Am J Obstet Gynecol 2017;217(04):425.e1-425.e16

56 Lum D, Guido R, Rodriguez E, et al. Brush cytology of the fallopian tube and implications in ovarian cancer screening. J Minim Invasive Gynecol 2014;21(05):851-856

57 La Vecchia C. Epidemiology of ovarian cancer: a summary review. Eur J Cancer Prev 2001;10(02):125-129

58 Reid BM, Permuth JB, Sellers TA. Epidemiology of ovarian cancer: a review. Cancer Biol Med 2017;14(01):9-32

59 Praestegaard C, Jensen A, Jensen SM, et al; Australian Ovarian Cancer Study Group; Ovarian Cancer Association Consortium. Cigarette smoking is associated with adverse survival among women with ovarian cancer: Results from a pooled analysis of 19 studies. Int J Cancer 2017;140(11):2422-2435

60 Seitz HK, Pelucchi C, Bagnardi V, La Vecchia C. Epidemiology and pathophysiology of alcohol and breast cancer: Update 2012. Alcohol Alcohol 2012;47(03):204-212

61 Chuffa LG, Fioruci-Fontanelli BA, Mendes LO, et al. Characterization of chemically induced ovarian carcinomas in an ethanolpreferring rat model: influence of long-term melatonin treatment. PLoS One 2013;8(12):e81676

62 Genkinger JM, Hunter DJ, Spiegelman D, et al. Alcohol intake and ovarian cancer risk: a pooled analysis of 10 cohort studies. $\mathrm{Br} \mathrm{J}$ Cancer 2006;94(05):757-762

$63 \mathrm{Kim}$ HS, Kim JW, Shouten LJ, et al. Wine drinking and epithelial ovarian cancer risk: a meta-analysis. J Gynecol Oncol 2010;21 (02):112-118

64 O'Hanlon LH. Studying the connection between exercise and cancer risk reduction. J Natl Cancer Inst 2013;105(11):753-754

65 Divella R, De Luca R, Abbate I, Naglieri E, Daniele A. Obesity and cancer: the role of adipose tissue and adipo-cytokines-induced chronic inflammation. J Cancer 2016;7(15):2346-2359

66 Nagle CM, Dixon SC, Jensen A, et al; Australian Ovarian Cancer Study Group; Ovarian Cancer Association Consortium. Obesity and survival among women with ovarian cancer: results from the Ovarian Cancer Association Consortium. Br J Cancer 2015;113 (05):817-826
67 Crane TE, Khulpateea BR, Alberts DS, Basen-Engquist K, Thomson CA. Dietary intake and ovarian cancer risk: a systematic review. Cancer Epidemiol Biomarkers Prev 2014;23(02):255-273

68 Huncharek M, Kupelnick B. Dietary fat intake and risk of epithelial ovarian cancer: a meta-analysis of 6,689 subjects from 8 observational studies. Nutr Cancer 2001;40(02):87-91

69 Kolahdooz F, Ibiebele TI, van der Pols JC, Webb PM. Dietary patterns and ovarian cancer risk. Am J Clin Nutr 2009;89(01): 297-304

70 Merritt MA, Tzoulaki I, van den Brandt PA, et al. Nutrient-wide association study of 57 foods/nutrients and epithelial ovarian cancer in the European Prospective Investigation into Cancer and Nutrition study and the Netherlands Cohort Study. Am J Clin Nutr 2016;103(01):161-167

71 Genkinger JM, Hunter DJ, Spiegelman D, et al. A pooled analysis of 12 cohort studies of dietary fat, cholesterol and egg intake and ovarian cancer. Cancer Causes Control 2006;17(03):273-285

72 Hou R, Wu QJ, Gong TT, Jiang L. Dietary fat and fatty acid intake and epithelial ovarian cancer risk: evidence from epidemiological studies. Oncotarget 2015;6(40):43099-43119

73 Turati F, Galeone C, Gandini S, et al. High glycemic index and glycemic load are associated with moderately increased cancer risk. Mol Nutr Food Res 2015;59(07):1384-1394

74 Kolahdooz F, van der Pols JC, Bain CJ, et al; Australian Cancer Study (Ovarian Cancer) and the Australian Ovarian Cancer Study Group. Meat, fish, and ovarian cancer risk: Results from 2 Australian case-control studies, a systematic review, and meta-analysis. Am J Clin Nutr 2010;91(06):1752-1763

75 Bar-Sela G, Epelbaum R, Schaffer M. Curcumin as an anti-cancer agent: review of the gap between basic and clinical applications. Curr Med Chem 2010;17(03):190-197

76 Basnet P, Skalko-Basnet N. Curcumin: an anti-inflammatory molecule from a curry spice on the path to cancer treatment. Molecules 2011;16(06):4567-4598

77 Bishayee A. Cancer prevention and treatment with resveratrol: from rodent studies to clinical trials. Cancer Prev Res (Phila) 2009; 2(05):409-418

78 Quoc Trung L, Espinoza JL, Takami A, Nakao S. Resveratrol induces cell cycle arrest and apoptosis in malignant NK cells via JAK2/ STAT3 pathway inhibition. PLoS One 2013;8(01):e55183

79 Amin A, Buratovich M. The anti-cancer charm of flavonoids: a cup-of-tea will do!. Recent Patents Anticancer Drug Discov 2007; 2(02):109-117

80 Chia VM, O'Malley CD, Danese MD, et al. Prevalence and incidence of comorbidities in elderly women with ovarian cancer. Gynecol Oncol 2013;129(02):346-352

81 Bakhru A, Buckanovich RJ, Griggs JJ. The impact of diabetes on survival in women with ovarian cancer. Gynecol Oncol 2011;121 (01):106-111

82 Minlikeeva AN, Freudenheim JL, Cannioto RA, et al; Australian Ovarian Cancer Study Group; Ovarian Cancer Association Consortium. History of hypertension, heart disease, and diabetes and ovarian cancer patient survival: evidence from the ovarian cancer association consortium. Cancer Causes Control 2017;28(05): 469-486

83 Sood AK, Bhatty R, Kamat AA, et al. Stress hormone-mediated invasion of ovarian cancer cells. Clin Cancer Res 2006;12(02): 369-375

84 Watkins JL, Thaker PH, Nick AM, et al. Clinical impact of selective and nonselective beta-blockers on survival in patients with ovarian cancer. Cancer 2015;121(19):3444-3451

85 Eskander R, Bessonova L, Chiu C, et al. Beta blocker use and ovarian cancer survival: a retrospective cohort study. Gynecol Oncol 2012;127:S21. Doi: 10.1016/j.ygyno.2012.07.059

86 Wang SB, Lei KJ, Liu JP, Jia YM. Continuous use of metformin can improve survival in type 2 diabetic patients with ovarian cancer: A retrospective study. Medicine (Baltimore) 2017;96 (29):e7605 
87 Huo J, Bian XH, Huang Y, Miao ZC, Song LH. Inhibitory effect and mechanism of metformin on human ovarian cancer cells SKOV-3 and A2780. Eur Rev Med Pharmacol Sci 2017;21(03):484-489

88 Fu YL, Zhang QH, Wang XW, He H. Antidiabetic drug metformin mitigates ovarian cancer SKOV3 cell growth by triggering G2/M cell cycle arrest and inhibition of m-TOR/PI3K/Akt signaling pathway. Eur Rev Med Pharmacol Sci 2017;21(05):1169-1175

89 Shen CJ, Chang CC, Chen YT, Lai CS, Hsu YC. Melatonin suppresses the growth of ovarian cancer cell lines (OVCAR-429 and PA-1) and potentiates the effect of G1 arrest by targeting CDKs. Int J Mol Sci 2016;17(02):176

$90 \mathrm{Li}$ Y, Li S, Zhou Y, et al. Melatonin for the prevention and treatment of cancer. Oncotarget 2017;8(24):39896-39921

91 Maruotti N, Cantatore FP. Vitamin D and the immune system. J Rheumatol 2010;37(03):491-495

92 Lurie G, Matsuno RK, Wilkens LR, et al. Cataract and ovarian carcinoma: is the vitamin D hypothesis alive? Cancer Epidemiol Biomarkers Prev 2011;20(12):2507-2511

93 Ross AC, Manson JE, Abrams SA, et al. The 2011 report on dietary reference intakes for calcium and vitamin $D$ from the Institute of
Medicine: what clinicians need to know. J Clin Endocrinol Metab 2011;96(01):53-58

94 Saunders DE, Christensen C, Wappler NL, et al. Inhibition of c-myc in breast and ovarian carcinoma cells by 1,25 -dihydroxyvitamin D3, retinoic acid and dexamethasone. Anticancer Drugs 1993; 4(02):201-208

95 Merritt MA, Cramer DW, Vitonis AF, Titus LJ, Terry KL. Dairy foods and nutrients in relation to risk of ovarian cancer and major histological subtypes. Int J Cancer 2013;132(05):1114-1124

96 Lungchukiet P, Sun Y, Kasiappan R, et al. Suppression of epithelial ovarian cancer invasion into the omentum by $1 \alpha, 25$-dihydroxyvitamin D3 and its receptor. J Steroid Biochem Mol Biol 2015; 148:138-147

97 Feldman D, Krishnan AV, Swami S, Giovannucci E, Feldman BJ. The role of vitamin $D$ in reducing cancer risk and progression. Nat Rev Cancer 2014;14(05):342-357

98 Liu L, Hu Z, Zhang H, et al. Vitamin D postpones the progression of epithelial ovarian cancer induced by 7, 12-dimethylbenz [a] anthracene both in vitro and in vivo. Onco Targets Ther 2016; 9:2365-2375 